\title{
Simulation of dendritic crystal growth with thermal convection
}

\author{
EBERHARD B ÄNSCH ${ }^{\dagger}$ \\ Zentrum für Technomathematik, Universität Bremen, FB 3, Postfach 330440, 28334 Bremen, \\ Germany \\ AND \\ ALFRED SCHMIDT \\ Institut für Angewandte Mathematik, Universität Freiburg, Hermann-Herder-Strasse 10, 79104 \\ Freiburg, Germany
}

[Received 15 March 1999 and in revised form 23 August 1999]

\begin{abstract}
The dendritic growth of crystals under gravity influence shows a strong dependence on convection in the liquid. The situation is modelled by the Stefan problem with a Gibbs-Thomson condition coupled with the Navier-Stokes equations in the liquid phase. A finite element method for the numerical simulation of dendritic crystal growth including convection effects is presented. It consists of a parametric finite element method for the evolution of the interface, coupled with finite element solvers for the heat equation and Navier-Stokes equations in a time dependent domain. Results from numerical simulations in two space dimensions with Dirichlet and transparent boundary conditions are included.
\end{abstract}

Keywords: Dendritic solidification; crystal growth; natural convection; Gibbs-Thomson; NavierStokes equations; finite elements

\section{Introduction}

When a small seed crystal is placed (or nucleates) in an undercooled melt, the solid phase grows rapidly. Directional anisotropies of surface and kinetic energies, due to the underlying molecular geometry, for example, result in preferred growth directions and the development of dendrites $[13,18]$. Experiments under $1 g$ earth gravity conditions show a strong dependence of the growth velocities and the resulting structures on the angle between the growth direction and gravity vector, especially for low undercooling [11]. These effects are attributed to (natural) thermal convection in the liquid, driven by buoyancy forces. Recent micro gravity (i.e. vanishing or nearly zero gravity) space shuttle experiments show similar effects that can also be ascribed to thermal convection and thus underline its importance [12].

Some theoretical investigations of dendritic growth with convection were performed for a special case, where the interface is parabolic, the main growth direction is parallel to the gravity vector, and the phase transition is modelled by the classical one-phase Stefan problem with an isothermal interface, without any surface tension or kinetic undercooling and without any anisotropy in the equations. Using a boundary layer flow model, the effect of fluid flow on steady dendrite growth

\footnotetext{
†Email: baensch@math.uni-bremen.de

†mail: alfred.schmidt@mathematik.uni-freiburg.de
} 
was studied by Cantor \& Vogel [8]. Ananth \& Gill [1,2] are able to predict experimentally observed growth characteristics quite well for a wide range of physical parameters.

Numerical simulations were done recently by Griebel et al. [16]. They consider an extended model with additional density changes and present a numerical algorithm based on a finite difference method and a surface tracking method to capture the phase boundaries.

We present a numerical algorithm based on a sharp interface model, i.e. the free boundary is assumed to be a smooth curve (but maybe with high curvature). Finite element approximations are used for temperature and velocity as well as for a parametrization of the moving interface. Adaptive methods based on local error indicators are used to generate locally refined meshes which allow for a high resolution of relevant data, especially near the interface.

The rest of this article is organized as follows: in Section 2 we present the equations, Section 3 deals with the finite element discretization and, finally, we present the numerical results with different convection strengths and boundary conditions in Section 4.

\section{The mathematical model}

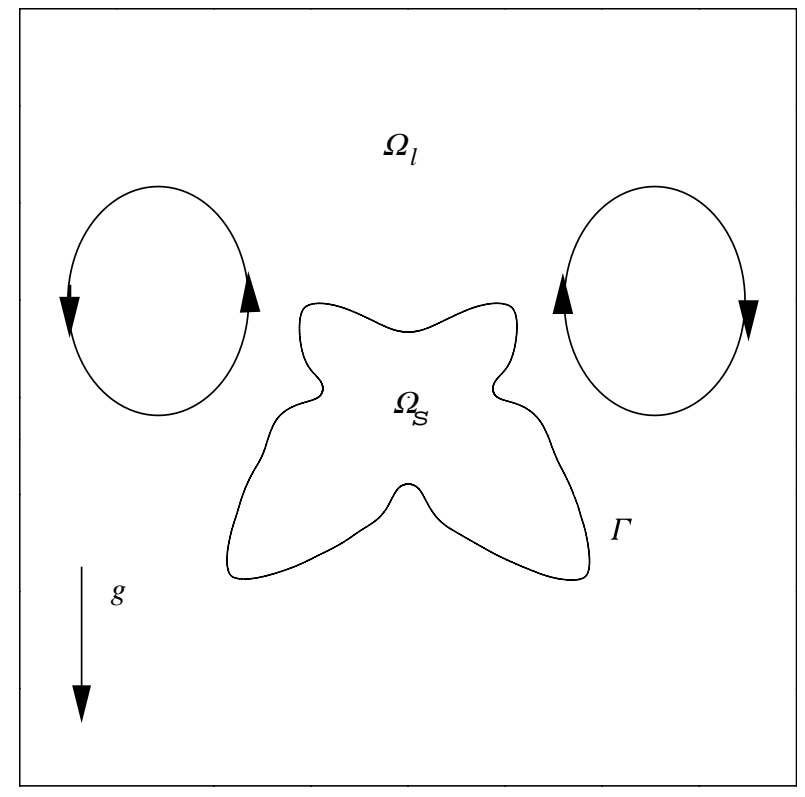

FIG. 1. Setting of the problem.

We consider a bounded container $\Omega \subset \mathbb{R}^{2}$ and an initial solid subdomain $\Omega_{s}(0) \subset \subset \Omega$ with solid-liquid interface $\Gamma(0)=\partial \Omega_{s}(0)$. The liquid subdomain is $\Omega_{l}(t)=\Omega \backslash \overline{\Omega_{s}(t)}$. The model includes the temperature $\vartheta$, velocity $u$, pressure $p$, and the time dependent distribution of phases with a moving solid-liquid interface $\Gamma$.

The heat equation (3), (4) models energy diffusion in both the liquid and solid, with an advection term in the liquid. The Stefan condition (5) and a Gibbs-Thomson condition (6) with anisotropic kinetic and surface terms model the phase transition. Here, $V_{\Gamma}$ and $C_{\Gamma}$ denote the 
scalar interface velocity and curvature, respectively, while $\epsilon_{V}, \epsilon_{C}$ are coefficient functions which depend on the interface normal $\nu_{\Gamma}$. If the surface energy anisotropy $\gamma(v)$ is smooth, and $\tilde{\gamma}(s):=$ $\gamma(\cos (s), \sin (s))=\gamma(v)$, then $\epsilon_{C}(v)=\tilde{\gamma}(s)+\tilde{\gamma}^{\prime \prime}(s)$. A generalization to (nearly) crystalline anisotropy is possible, compare [21]. We use an additional coefficient $C_{c o n v}$ to model different strengths of advection.

The Boussinesq approximation with gravity vector $g=-e_{2}=(0,-1)^{T}$ is used to account for the buoyancy forces (1), and the liquid is assumed to be incompressible (2). We assume that the material density is constant and does not change between the solid and liquid. This leads to a nonslip boundary condition on the interface (7). A density change between the solid and liquid would induce a normal velocity $u \cdot v_{\Gamma}$ proportional to the interface velocity $V_{\Gamma}$, compare [16].

After an appropriate scaling, introducing the Grashof number $G r$, Prandtl number $P r$, and scaled latent heat $L$, the dimensionless equations read:

$$
\begin{aligned}
\frac{\partial u}{\partial t}+u \cdot \nabla u-\frac{1}{\sqrt{G r}} \Delta u+\nabla p & =\vartheta e_{2} & & \text { in } \Omega_{l}, \\
\nabla \cdot u & =0 & & \text { in } \Omega_{l} . \\
\frac{\partial \vartheta}{\partial t}+C_{\text {conv }} u \cdot \nabla \vartheta-\frac{1}{\operatorname{Pr} \sqrt{G r}} \Delta \vartheta & =0 & & \text { in } \Omega_{l}, \\
\frac{\partial \vartheta}{\partial t}-\frac{1}{\operatorname{Pr} \sqrt{G r}} \Delta \vartheta & =0 & & \text { in } \Omega_{s} . \\
{\left[\frac{1}{\operatorname{Pr} \sqrt{G r}} \partial_{v} \vartheta\right]+L V_{\Gamma} } & =0 & & \text { on } \Gamma, \\
\epsilon_{V} V_{\Gamma}+\epsilon_{C} C_{\Gamma}+\vartheta & =0 & & \text { on } \Gamma, \\
u & =0 & & \text { on } \Gamma .
\end{aligned}
$$

In the Stefan condition (5), $[\cdot]:=(\cdot)_{\text {liquid }}-(\cdot)_{\text {solid }}$ denotes the jump across the interface. The system is completed by initial and boundary conditions:

$$
\begin{array}{rlrl}
u(\cdot, 0) & =0 & & \text { in } \Omega, \\
u(\cdot, t)=0 & & \text { on } \partial \Omega_{l}, \\
\vartheta(\cdot, 0) & =\vartheta_{0} & & \text { in } \Omega, \\
\vartheta(\cdot, t) & =\vartheta_{0} & & \text { on } \partial \Omega, \\
\Gamma(0) & =\Gamma_{0} . & &
\end{array}
$$

With appropriately low initial temperature data $\vartheta_{0}$, the liquid is undercooled, and dendritic growth of the solid phase is expected. 


\section{Weak formulations and discretization}

\subsection{Heat equation with Stefan and Gibbs-Thomson conditions}

Following the lines of $[6,20,21]$, we divide problem (3)-(6) into an anisotropic mean curvature flow equation for $\Gamma$ and a modified heat equation. The weak formulation and discretization of the interface motion uses the approach of Dziuk [9] to mean curvature evolution of curves and surfaces.

Using an extension $u=0$ in $\Omega_{s}$, we multiply (3) and (4) with a test function $\phi \in H^{1}(\Omega)$, use integration by parts in both subdomains, and replace the occurring jump of normal derivatives by the Stefan and Gibbs-Thomson conditions to arrive at

$$
\int_{\Omega}\left(\frac{\partial \vartheta}{\partial t} \phi+C_{\text {conv }} u \cdot \nabla \vartheta \phi+\frac{1}{P r \sqrt{G r}} \nabla \vartheta \cdot \nabla \phi\right)+\int_{\Gamma} \frac{L}{\epsilon_{V}} \vartheta \phi=-\int_{\Gamma} \frac{L \epsilon_{C}}{\epsilon_{V}} C_{\Gamma} \phi
$$

for all $\phi$. For the interface motion, the Gibbs-Thomson condition (6) leads to a weak formulation that is similar to a heat equation. To begin, the curvature vector $C_{\Gamma} v$ can be written as the LaplaceBeltrami operator applied to the identity id $: \Gamma \rightarrow \mathbb{R}^{2}$, or to the parametrization $x: \Gamma \rightarrow \mathbb{R}^{2}$. After multiplication with a test function $\psi \in H^{1}(\Gamma)$ and integration by parts, where $\bar{\nabla}$ denotes the tangential (covariant) derivative, we obtain:

$$
\int_{\Gamma}\left(\frac{\epsilon_{V}}{\epsilon_{C}} \frac{\partial x}{\partial t} \psi+\bar{\nabla} x \cdot \bar{\nabla} \psi\right)=-\int_{\Gamma} \frac{1}{\epsilon_{C}} v \vartheta \psi \quad \text { for all } \psi .
$$

Assuming that $\Gamma$ is a closed Lipschitz curve or hypersurface, all terms are well defined and no boundary integrals appear from the integration by parts.

Both weak formulations are well suited for finite element discretizations. After a time discretization with time steps $t_{0}=0<t_{1}<t_{2}<\cdots$ and time step sizes $\tau_{n}=t_{n}-t_{n-1}$, we use conforming subdivisions $\mathcal{T}^{n}$ of $\Omega$ into triangles and corresponding spaces $\mathcal{V}^{n}$ of piecewise quadratic finite element functions over $\mathcal{T}^{n}$ and $\mathcal{V}_{0}^{n}:=\mathcal{V}^{n} \cap H_{0}^{1}(\Omega)$. Given an approximation $\Theta^{0} \in \mathcal{V}_{0}^{0}$ of $\vartheta_{0}$, discrete temperatures $\Theta^{n+1} \in \mathcal{V}^{n+1}$ are defined for $n \geqslant 0$ by

$$
\begin{gathered}
\int_{\Omega}\left(\frac{\Theta^{n+1}-\Theta^{n}}{\tau_{n+1}} \Phi+C_{\text {conv }} u \cdot \nabla \Theta^{n+1} \Phi+\frac{1}{\operatorname{Pr} \sqrt{G r}} \nabla \Theta^{n+1} \cdot \nabla \Phi\right) \\
+\int_{\Gamma^{n+1}} \frac{L}{\epsilon_{V}} \Theta^{n+1} \Phi=-\int_{\Gamma^{n+1}} \frac{\epsilon_{C} L}{\epsilon_{V}} C_{\Gamma^{n+1}} \Phi \quad \text { for all } \Phi \in \mathcal{V}_{0}^{n+1}
\end{gathered}
$$

with appropriate Dirichlet boundary conditions on $\partial \Omega$. By using the Gibbs-Thomson relation, we obtain an implicit term for $\Theta^{n+1}$ on the free boundary $\Gamma^{n+1}$ on the left-hand side, which is positive, semi-definite, and additionally leads to a good approximation for the temperature on the interface. Such values will be used on the right-hand side of the interface propagation equation (11) below.

Using a polygonal, piecewise quadratic discretization of the interface $\Gamma^{n}$ and isoparametric finite element spaces $\mathcal{W}^{n}$ over $\Gamma^{n}$, we define parametrizations $X^{n+1} \in\left(\mathcal{W}^{n}\right)^{2}: \Gamma^{n} \rightarrow \mathbb{R}^{2}$ of $\Gamma^{n+1}$ by

$$
\int_{\Gamma^{n}}\left(\frac{\epsilon_{V}}{\epsilon_{C}} \frac{X^{n+1}-i d}{\tau_{n+1}} \Psi+\bar{\nabla} X^{n+1} \cdot \bar{\nabla} \Psi\right)=-\int_{\Gamma^{n}} \frac{1}{\epsilon_{C}} v \Theta^{n} \Psi \quad \text { for all } \Psi \in \mathcal{W}^{n}
$$


and define the discrete interfaces by

$$
\Gamma^{n+1}:=X^{n+1}\left(\Gamma^{n}\right) .
$$

Finally, the curvature approximation $C_{\Gamma} \in \mathcal{W}^{n+1}$ is defined by first calculating a vector valued curvature $\vec{C}_{\Gamma} \in\left(\mathcal{W}^{n+1}\right)^{2}$ solving

$$
\int_{\Gamma^{n+1}} \vec{C}_{\Gamma} \Psi=\int_{\Gamma^{n+1}} \bar{\nabla} i d \cdot \bar{\nabla} \Psi \quad \text { for all } \Psi \in \mathcal{W}^{n+1} .
$$

The values of $C_{\Gamma}$ in vertices and edge midpoints of $\Gamma^{n+1}$ are defined as the scalar products $C_{\Gamma}(p)=\vec{C}_{\Gamma}(p) \cdot n_{p}$ where $n_{p}$ is an outer normal vector to $\Gamma^{n+1}$ at $p$, defined as a weighted sum of adjacent normals for the case that $p$ is a vertex, where the discrete interface is not smooth.

Note that by this approach we restrict ourselves to the case where no topological changes of liquid/solid occur. In particular, the solid region $\Omega_{s}(t) \subset \subset \Omega$ is bounded by the embedded curve $\Gamma(t) \subset \subset \Omega$ during the whole time interval (without any self-intersection or intersection with $\partial \Omega$ ). We will see in the numerical simulations that this is no real restriction, at least for moderate time intervals and in the range of parameters that we currently use. The only true restriction is an upper bound for the time interval given by the fact that the interface must not meet the boundary $\partial \Omega$.

The numerical approximation of dendritic growth (without convection) with this method gives much better results when piecewise quadratic finite element functions are used instead of piecewise linears. This holds especially for the interface and curvature approximation, compare [20].

Recently, Veeser [22, 23] proved convergence and error estimates for a semi-discrete finite element method for dendritic growth with a slightly different discretization of the mean curvature evolution.

\subsection{Navier-Stokes flow in a time dependent domain}

One particular problem in computing the flow field by using equations (1)-(2) is the time dependent definition of $\Omega_{l}(t)$. There are several ways to solve this problem in the discrete case, for instance:

- An explicit definition for $\Omega_{h, l}\left(t_{n}\right)$ and a no-slip condition for $u$ on $\partial \Omega_{h, l}\left(t_{n}\right)$.

- Fictitious domain approaches, where the Navier-Stokes equations are solved in the whole domain $\Omega$ and the no-slip boundary condition on $\Gamma$ is enforced only in a weak sense:

- A penalty approach, using an additional term $1 / \delta \int_{\Gamma^{n+1}} u \cdot \varphi$ in the corresponding bilinear form in the momentum equation, where $1 / \delta \gg 1$ is the penalty parameter.

- An implementation of the no-slip boundary condition on $\Gamma$ by an additional constraint, see, for instance, $[14,15]$.

Numerical experiments with all three methods above gave comparable solutions, but the first method turned out to be the most robust and by far, most efficient one. Therefore we will only address this method here. In order to apply this method we use the following definition for $\Omega_{h, l}\left(t_{n}\right)$ : an element $T \in \mathcal{T}^{n}$ is called liquid iff $T$ lies completely in the liquid region, i.e. all $x \in T$ lie outside the solid region defined by the curve $\Gamma^{n}$. It is called solid iff it is not liquid. Define

$$
\Omega_{h, l}\left(t_{n}\right):=\cup\left\{T \in \mathcal{T}^{n} \mid T \text { is liquid }\right\},
$$

see Fig. 2. An algorithm to mark all mesh elements as either liquid or solid is the following: 
- Follow the interface curve and mark all triangles $T$ with $T \cap \Gamma \neq \emptyset$ as solid.

- Mark all non-solid elements at $\partial \Omega$ as liquid.

- Continue by marking all non-solid neighbors of liquid elements as liquid, and repeat this step as long as possible.

- Finally, all not yet marked elements are solid elements.

This algorithm can be implemented easily with a computational complexity that is linear in the number of mesh elements.

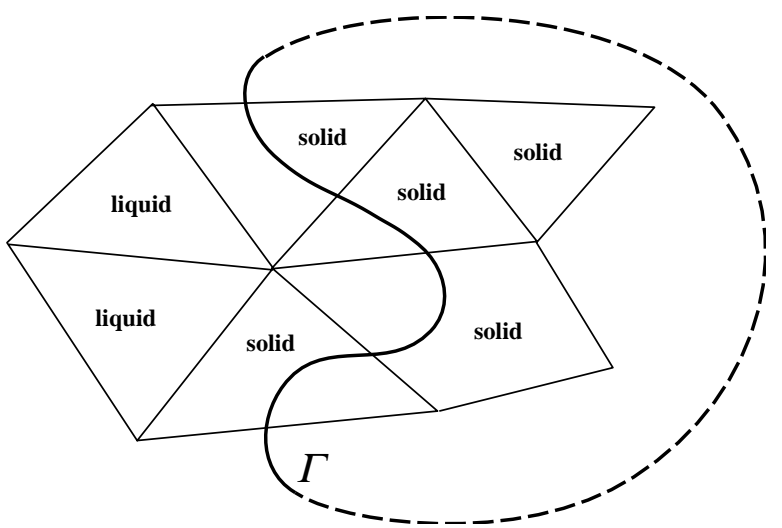

FIG. 2. Liquid and solid triangles.

To solve the Navier-Stokes equations we use a time discretization based on the fractional $\theta$ scheme in a variant as an operator splitting, which was proposed in [7]. For the space discretization, the Taylor-Hood element, i.e. piecewise quadratics for the velocity and piecewise linears for the pressure, is used. More precisely, let $\mathcal{Y}^{n}:=\left\{\Phi \in\left(\mathcal{V}_{0}^{n}\right)^{2} \mid \Phi=0\right.$ on $\left.\Omega \backslash \Omega_{h, l}^{n}\right\}$ be the space of globally continuous, vector valued piecewise quadratics on $\Omega_{h, l}^{n}:=\Omega_{h, l}\left(t_{n}\right)$, vanishing on $\partial \Omega_{h, l}^{n}$, and for convenience extended to $\Omega \backslash \Omega_{h, l}^{n}$ by zero. Likewise, $\mathcal{Z}^{n}$ denotes the space of globally continuous, piecewise linears on $\Omega_{h, l}^{n}$.

Let $\theta=1-\frac{\sqrt{2}}{2}$ and $\alpha, \beta \in(0,1), \alpha+\beta=1, \alpha>\frac{1}{2}$. Split each time interval $\left[t_{n}, t_{n+1}\right]$ into three subintervals $\left[t_{n}, t_{n}+\theta \tau_{n+1}\right],\left[t_{n}+\theta \tau_{n+1}, t_{n}+\theta^{\prime} \tau_{n+1}\right]$, and $\left[t_{n}+\theta^{\prime} \tau_{n+1}, t_{n+1}\right]$ with $\theta^{\prime}=1-\theta$. The fractional $\theta$-scheme applied to our case reads: for $n \geqslant 0$ find $U^{n+\theta}, U^{n+\theta^{\prime}}, U^{n+1} \in \mathcal{Y}^{n+1}$ and $P^{n+\theta}, P^{n+1} \in \mathcal{Z}^{n+1}$ such that

$$
U^{0}=0 \quad \text { in } \Omega
$$

and for all $\Phi \in \mathcal{Y}^{n+1}, \Psi \in \mathcal{Z}^{n+1}$ 


$$
\left\{\begin{array}{l}
\int_{\Omega_{h, l}^{n+1}}\left(\frac{U^{n+\theta}-\pi_{n+1} U^{n}}{\theta \tau_{n+1}} \Phi+\frac{\alpha}{\sqrt{G r}} \nabla U^{n+\theta} \nabla \Phi-P^{n+\theta} \nabla \cdot \Phi\right) \\
=\int_{\Omega_{h, l}^{n+1}}\left(\frac{-\beta}{\sqrt{G r}} \nabla U^{n} \nabla \Phi-\left(U^{n} \cdot \nabla\right) U^{n} \Phi+\Theta^{n+1} e_{2} \cdot \Phi\right) \\
\int_{\Omega_{h, l}^{n+1}} \nabla \cdot U^{n+\theta} \Psi=0
\end{array}\right.
$$

$$
\left\{\begin{array}{c}
\int_{\Omega_{h, l}^{n+1}}\left(\frac{U^{n+\theta^{\prime}}-U^{n+\theta}}{(1-2 \theta) \tau_{n+1}} \Phi+\frac{\beta}{\sqrt{G r}} \nabla U^{n+\theta^{\prime}} \nabla \Phi+\left(U^{n+\theta^{\prime}} \cdot \nabla\right) U^{n+\theta^{\prime}} \Phi\right) \\
=\int_{\Omega_{h, l}^{n+1}}\left(-\frac{\alpha}{\sqrt{G r}} \nabla U^{n+\theta} \nabla \Phi+P^{n+\theta} \nabla \cdot \Phi+\Theta^{n+1} e_{2} \cdot \Phi\right),
\end{array}\right.
$$

$$
\left\{\begin{array}{l}
\int_{\Omega_{h, l}^{n+1}}\left(\frac{U^{n+1}-U^{n+\theta^{\prime}}}{\theta \tau_{n+1}} \Phi+\frac{\alpha}{\sqrt{G r}} \nabla U^{n+1} \nabla \Phi-P^{n+1} \nabla \cdot \Phi\right) \\
\quad=\int_{\Omega_{h, l}^{n+1}}\left(\frac{-\beta}{\sqrt{G r}} \nabla U^{n+\theta^{\prime}} \nabla \Phi-\left(U^{n+\theta^{\prime}} \cdot \nabla\right) U^{n+\theta^{\prime}} \Phi+\Theta^{n+1} e_{2} \cdot \Phi\right), \\
\int_{\Omega_{h, l}^{n+1}} \nabla \cdot U^{n+\theta} \Psi=0 .
\end{array}\right.
$$
$\mathcal{Y}^{n+1}$

In (13), $\pi_{n+1}$ denotes a projection operator from $\mathcal{Y}^{n}$ to the discretely solenoidal functions in

$$
\pi_{n+1}: \mathcal{Y}^{n} \rightarrow \mathcal{Y}_{0}^{n+1}:=\left\{\Phi \in \mathcal{Y}^{n+1} \mid \int_{\Omega_{h, l}^{n+1}} \nabla \cdot \Phi \Psi=0 \quad \text { for all } \Psi \in \mathcal{Z}^{n+1}\right\}
$$

This projection is used in order to prevent spurious pressure spikes in regions where the mesh changes from $t_{n}$ to $t_{n+1}$, see Section 3.5 below. 
By scheme (13)-(15), two major numerical difficulties of the Navier-Stokes equations, the treatment of the solenoidal condition and the nonlinearity, are decoupled. In (13) and (15), one has to solve a linear, self-adjoint Stokes-like system, where the non-linearity is treated explicitly. The nonlinear part (14) is a Burger's-like system of equations, the divergence free condition is dropped and the pressure gradient is taken from the previous time step. Thus, by this operator splitting, one reduces the Navier-Stokes equations to two considerably simpler subproblems.

The Stokes-like subproblems are solved by a preconditioned conjugate gradient (CG) method applied to the Schur complement operator for $P$ and a non-linear generalized minimal residual (GMRES) solver is used for the Burger's problem, see [5] for details.

\subsection{Coupling of equations}

The overall numerical method is based on a semi-implicit time discretization scheme with a GaußSeidel-type coupling of the three subproblems of interface, temperature, and velocity evolution. In each time step:

(A) Solve one time step of the mean curvature flow equation (11) for the parametric interface, giving $\Gamma^{n+1}$. The temperature $\Theta^{n}$ is taken explicitly from the last time step.

(B) Using the old velocity field $U^{n}$ and the new interface $\Gamma^{n+1}$, solve one time step for the heat equation with Stefan-condition on the phase boundary (10), giving $\Theta^{n+1}$.

(C) Using the new temperature and interface (and thus the new liquid domain $\Omega_{h, l}^{n+1}$ ), solve one time step for the Navier-Stokes equation (13)-(15), giving $U^{n+1}$ and $P^{n+1}$.

We like to remark that for this semi-implicit coupling, no stability problems were observed experimentally. This means that we do not have to fulfill a Courant-Friedrichs-Lewy (CFL) condition which would cause very small time steps due to the highly refined grids.

\subsection{Initial values}

For the first time step, values of the initial temperature on the initial interface are used in step (A) as the driving force for the interface evolution. In order to obtain compatible temperature values for this problem, the initial temperature $\Theta^{0} \in \mathcal{V}^{0}$ is defined as the solution to a stationary problem similar to the time dependent one, compare [20]. In particular, we solve

$$
\int_{\Omega} \frac{1}{\operatorname{Pr} \sqrt{G r}} \nabla \Theta^{0} \cdot \nabla \Phi+\int_{\Gamma^{0}} \frac{L}{\epsilon_{V}} \Theta^{0} \Phi=-\int_{\Gamma^{0}} \frac{\epsilon_{C} L}{\epsilon_{V}} C_{\Gamma} \Phi \quad \text { for all } \Phi \in \mathcal{V}_{0}^{0}
$$

with Dirichlet boundary values on $\partial \Omega$ and given initial interface $\Gamma^{0}$. The implicit treatment of $\Theta^{0}$ on $\Gamma^{0}$ guarantees that the initial temperature is compatible. The initial mesh $\mathcal{T}^{0}$ and finite element space $\mathcal{V}^{0}$ are adapted to the initial temperature in the same way as described in the next section.

\subsection{Adaptive finite element method}

During the evolution, the interface grows a lot in length and complexity. Thus, an adaptive discretization of the interface is indispensable. Here, we use a simple adaptivity criterion, namely 
an upper limit for the length of each segment of the interface discretization. Every segment which grows longer than a given tolerance is refined by bisection at its midpoint. Such refinements are done in each time step at the beginning of part (A), before calculating the new interface.

The same triangulations $\mathcal{T}^{n}$ of the domain $\Omega$ are used to define finite element spaces for discrete temperature and velocity. Local refinement of the meshes is based on a posteriori control of the discrete temperature. Numerical tests show that it is not necessary to use a combination of error estimators for temperature and velocity, as the velocity is much smoother than the temperature.

The Stefan condition (5) for the temperature implies a jump in the normal derivatives of $\vartheta$ at $\Gamma$. Moreover, steep gradients of $\vartheta$ occur close to the free boundary $\Gamma$. In order to approximate such a temperature field sufficiently well, we use adaptively refined meshes. Using highly refined meshes at $\Gamma$ one also reduces the 'roughness' of $\partial \Omega_{h, l}$.

Following the lines of $[10,20]$, we use a posteriori error indicators which can be computed from the discrete temperature and given data for the heat equation. Assuming regularity of the temperature $\vartheta(\cdot, t) \in H^{1, \infty}(\Omega) \cap H^{2,2}\left(\Omega_{l} \cup \Omega_{s}\right)$ with corresponding a priori estimates, the usual derivation of residual a posteriori error estimates leads to local error indicators

$$
\eta_{T}(\Theta)^{2}= \begin{cases}h_{T}^{4}\left\|\partial_{t} \Theta+C_{\text {conv }} u \cdot \nabla \Theta-\frac{\Delta \Theta}{\operatorname{Pr} \sqrt{G r}}\right\|_{L^{2}(T)}^{2} & \text { if } T \cap \Gamma=\emptyset, \\ +h_{T}^{3}\left\|\frac{1}{\operatorname{Pr} \sqrt{G r}}\left[\frac{\partial \Theta}{\partial v}\right]\right\|_{L^{2}(\partial T \backslash \partial \Omega)}^{2}\left\|{ }_{T}^{3}\right\| \partial_{t} \Theta+C_{\text {conv }} u \cdot \nabla \Theta-\frac{\Delta \Theta}{\operatorname{Pr} \sqrt{G r}} \|_{L^{2}(T)}^{2} & \\ +h_{T}^{2}\left\|\frac{L}{\epsilon_{V}}\left(\Theta+\epsilon_{C} C_{\Gamma}\right)\right\|_{L^{2}(\Gamma \cap T)}^{2} & \\ +h_{T}^{2}\left\|\frac{1}{\operatorname{Pr} \sqrt{G r}}\left[\frac{\partial \Theta}{\partial v}\right]\right\|_{L^{2}(\partial T \backslash \partial \Omega)}^{2} & \text { otherwise }\end{cases}
$$

for all $T \in \mathcal{T}$, where $\partial_{t} \Theta$ denotes the temporal difference quotient and $\left[\frac{\partial \Theta}{\partial \nu}\right]$ the jump of normal derivatives over inner edges of the triangulation. The lower $h$ exponents near $\Gamma$ account for the loss of regularity of the temperature at the free boundary. In part (B) of each time step, the triangulation is adapted by local refinement and coarsening of mesh elements such that the indicators $\eta_{T}(\Theta)^{2}$ are (nearly) equidistributed over all elements and the total estimate $\left(\sum_{T \in \mathcal{T}} \eta_{T}(\Theta)^{2}\right)^{1 / 2}$ is smaller than a given error tolerance. We use a semi-implicit adaptive method [4]; in particular, a solution $\tilde{\Theta}^{n+1} \in \mathcal{V}^{n}$ is calculated on the old mesh, then the mesh is adapted using indicators $\eta_{T}\left(\tilde{\Theta}^{n+1}\right)$, and finally $\Theta^{n+1} \in \mathcal{V}^{n+1}$ is computed on the new mesh. For the refinement and coarsening of triangular meshes we use algorithms based on the bisection of elements [3, 19].

As indicated, the same meshes are used for velocity discretization. Unfortunately, mesh changes between time steps which imply changing velocity spaces may introduce an inconsistency in the discrete Navier-Stokes equation due to the violation of the discrete solenoidal condition. The use of a simple nodal interpolation, say $I_{n+1} U^{n}$ instead of $\pi_{n+1} U^{n}$ in (13), would add a term

$$
\frac{1}{\theta \tau_{n}} I_{n+1} U^{n} \notin \mathcal{Y}_{0}^{n+1},
$$

which is not discretely divergence free. This would lead to strong numerical oscillations in the pressure $P$. Therefore we use a projection $\pi_{n+1}$, defined by: for $U \in \mathcal{Y}^{n}$ find $\left(\pi_{n+1} U, q\right) \in \mathcal{Y}^{n+1} \times$ 
$\mathcal{Z}^{n+1}$ such that for all $(\Phi, \Psi) \in \mathcal{Y}^{n+1} \times \mathcal{Z}^{n+1}$

$$
\begin{aligned}
\int_{\Omega_{h, l}^{n+1}}\left(\gamma_{1} \pi_{n+1} U \Phi+\gamma_{2} \nabla\left(\pi_{n+1} U\right) \nabla \Phi-q \nabla \cdot \Phi\right) & =\int_{\Omega_{h, l}^{n+1}}\left(\gamma_{1} U \Phi+\gamma_{2} \nabla U \nabla \Phi\right), \\
\int_{\Omega_{h, l}^{n+1}} \nabla \cdot\left(\pi_{n+1} U\right) \Psi & =0
\end{aligned}
$$

with $\gamma_{1}, \gamma_{2}>0$. This means that $\pi_{n+1}$ is a weighted $H^{1}$-projection to the space of discretely divergence free functions $\mathcal{Y}_{0}^{n+1}$. Note that $\pi_{n+1}=i d$ if $\left\{T \in \Omega_{h, l}^{n+1} \mid T\right.$ is liquid $\}=\{T \in$ $\Omega_{h, l}^{n} \mid T$ is liquid\}, i. e. no refinement or coarsening was done in the liquid subdomain.

\section{Numerical Simulations}

We consider problem (1)-(7) in the domain $\Omega=(-8,+8)^{2}$ with Grashof number $G r=100$, Prandtl number $\operatorname{Pr}=0.1$, latent heat $L=1$, and anisotropy functions

$$
\epsilon_{C}(\cos s, \sin s)=0.001+0.0003 \cos (4 s), \quad \epsilon_{V}(\cos s, \sin s)=0.01+0.003 \cos (4 s) .
$$

The initial interface $\Gamma_{0}$ is a circle of radius 0.05 with center $(0,0)$. Boundary values for the temperature are chosen as the external undercooling $\vartheta=-0.5$ on $\partial \Omega$; initial temperature is then given by (16). Finite element computations use piecewise quadratic approximations of $u, \vartheta$, and $\Gamma$. The meshes are adapted using the a posteriori techniques described in Section 3.5, and the time step size is fixed for all computations to $\tau=0.01$.

\subsection{Dirichlet problem}

We want to demonstrate the influence of the parameter $C_{\text {conv }}$ in a Dirichlet environment. Boundary values are

$$
\vartheta=-0.5, \quad u=0 \quad \text { on } \partial \Omega .
$$

The following figures compare results for $C_{\text {conv }} \in\{0,10,100\}$. For $C_{c o n v}=0$, the convection has no influence on the heat equation and on the phase transition; this case describes crystal growth under zero gravity with diffusion only. For $C_{c o n v}=10$ or 100 , the influence of the additional advection in the heat equation is clearly visible. Due to the convection in the liquid, the latent heat set free during solidification is transported away from the lower dendrite branches more quickly. This results in larger growth velocities for the lower branches of the crystal.

Figures 4-6 show the interfaces after $0,20, \ldots, 400$ time steps at times $t=k 0.2, k=$ $0,1,2, \ldots, 20$. While the upper and lower dendrite branches are symmetric for $C_{c o n v}=0$, the lower branches are faster than the upper ones for $C_{c o n v}>0$. It can be seen that, with strong convection, both upper and lower branches may be faster than in the diffusion-only case.

The three pictures in Fig. 3 show the velocities of dendrite tips from the three simulations. The faster tip velocities correspond to the lower dendrite branches. 

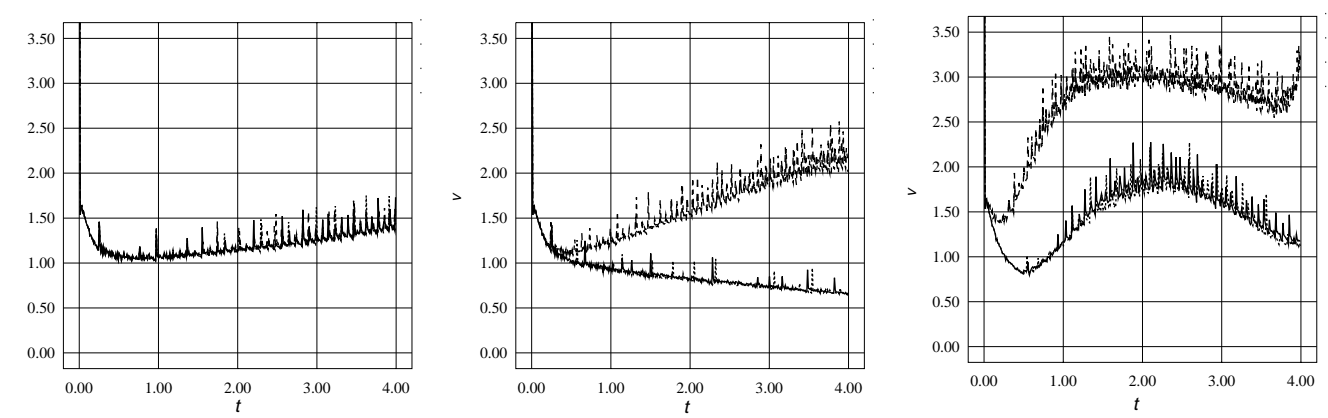

FIG. 3. Problem 4.1, $C_{\text {conv }}=0,10,100$; tip velocities.

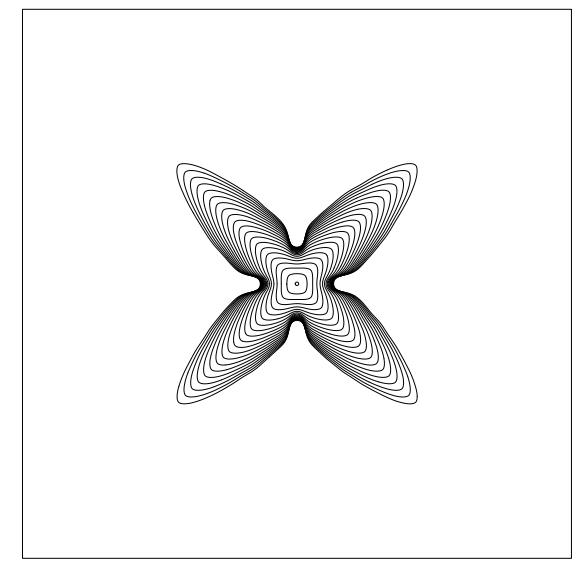

FIG. 4. Problem 4.1, $C_{\text {conv }}=0$; interfaces at $t=0.0,0.2, \ldots, 4.0$.

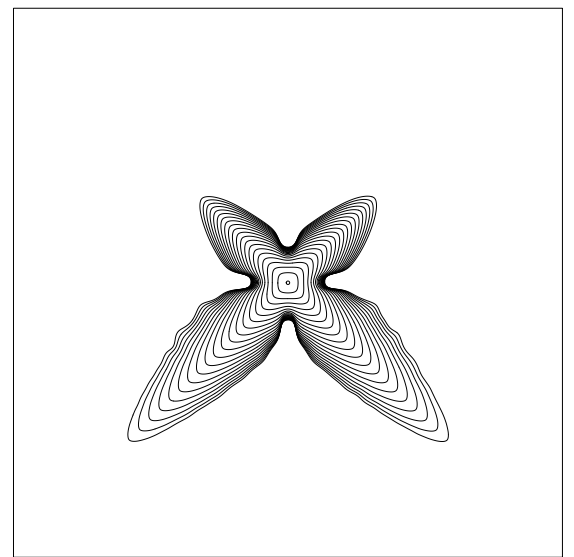

FIG. 5. Problem 4.1, $C_{\text {conv }}=10$; interfaces at $t=0.0,0.2, \ldots, 4.0$. 


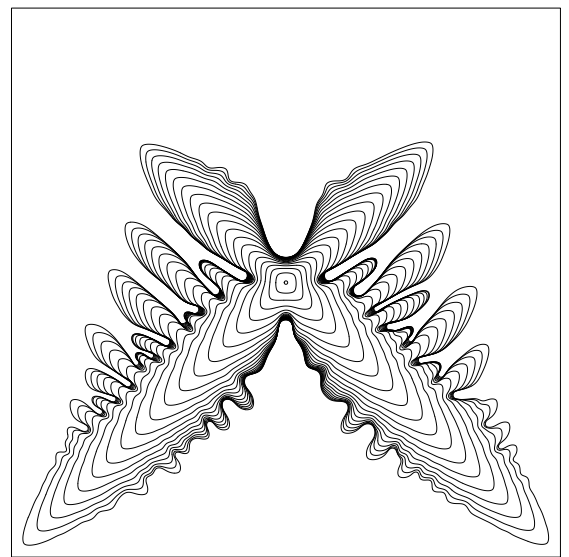

FIG. 6. Problem 4.1, $C_{c o n v}=100$; interfaces at $t=0.0,0.2, \ldots, 4.0$.

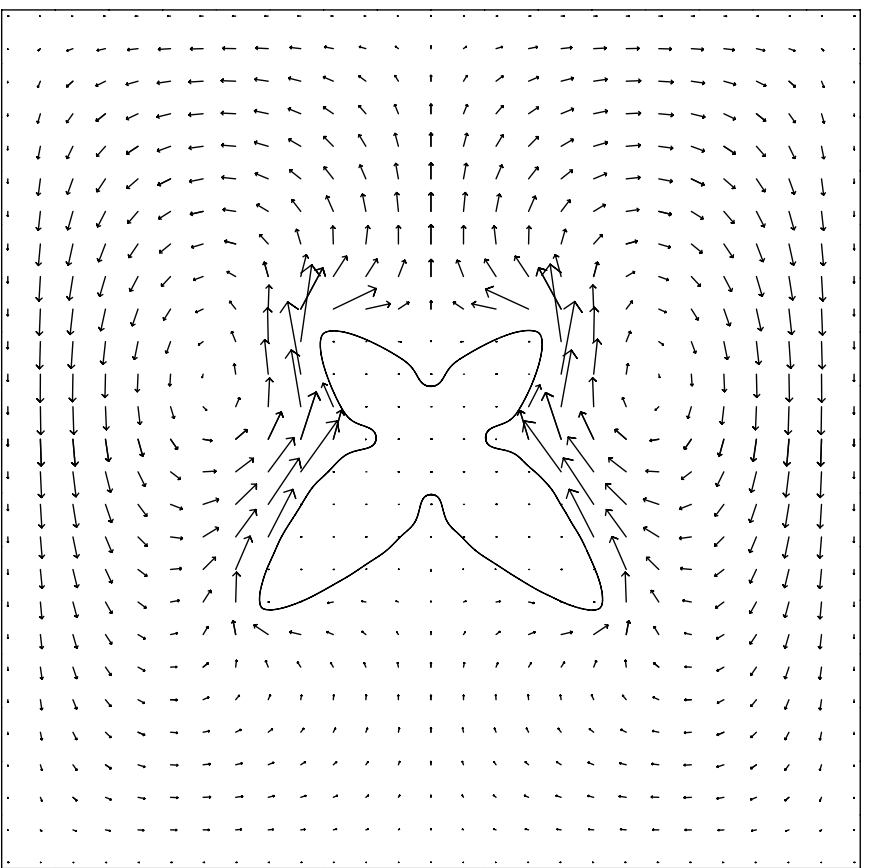

FIG. 7. Problem 4.1, $C_{\text {conv }}=10$; velocity at $t=3.0$. 


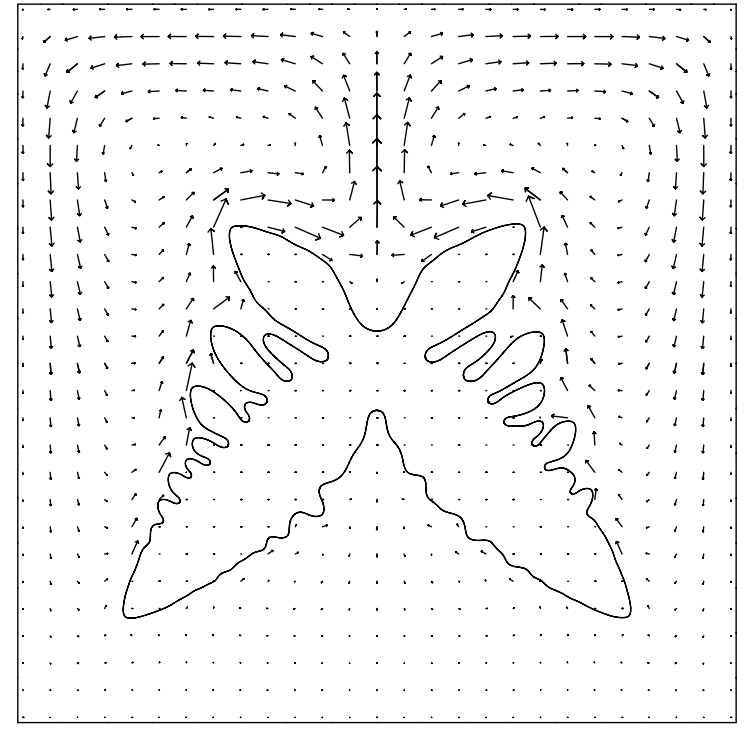

FIG. 8. Problem 4.1, $C_{c o n v}=100$; velocity at $t=3.0$.

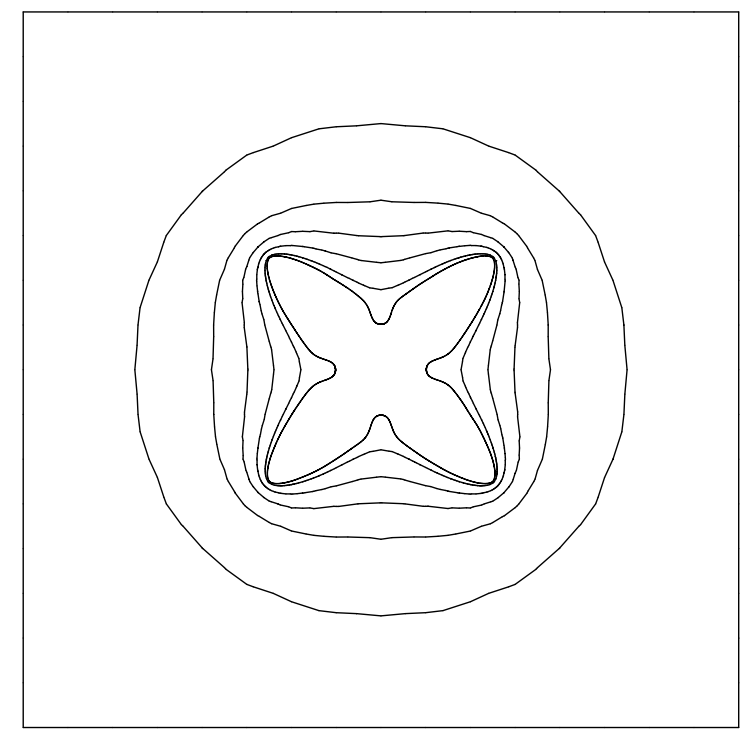

FIG. 9. Problem 4.1, $C_{\text {conv }}=0$; isothermal lines at $t=3.0$.

Velocities and interfaces at $t=3.0$ are shown in Figs 7 and 8. The maximal velocities, $\|V\|_{\infty}=$ 0.34 and 0.24 , respectively, are attained near the upper dendrite tips. Figures 9-11 show interfaces 


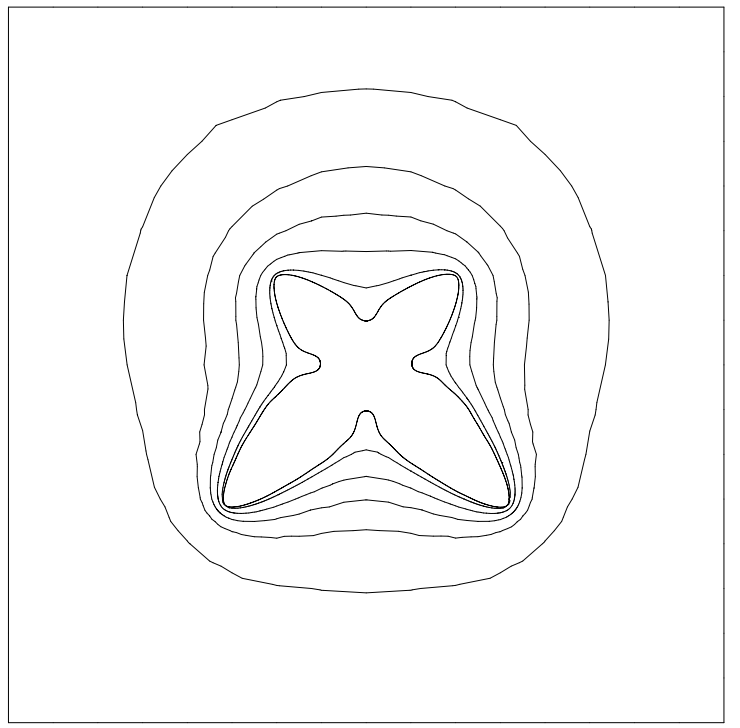

FIG. 10. Problem 4.1, $C_{\text {conv }}=10$; isothermal lines at $t=3.0$.

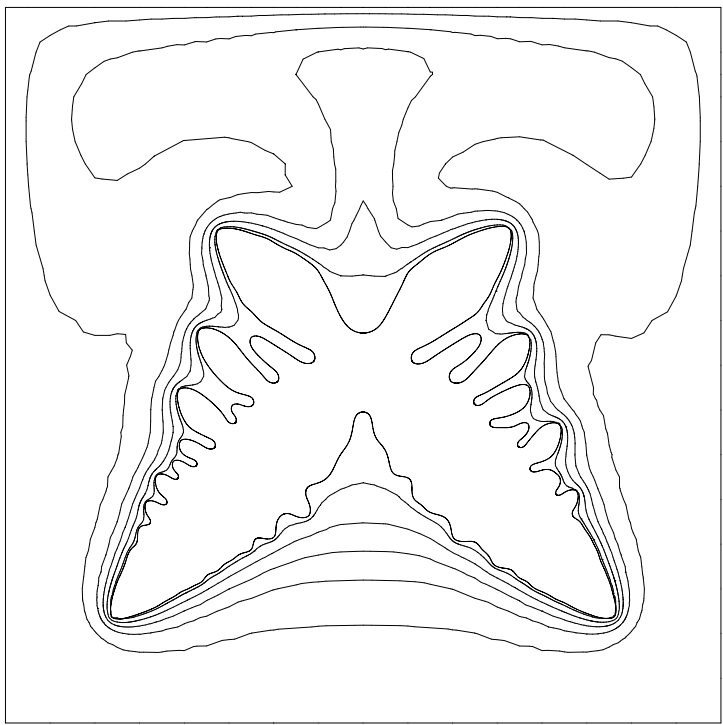

FIG. 11. Problem 4.1, $C_{c o n v}=100$; isothermal lines at $t=3.0$.

and isothermal lines $\Theta=-0.05,-0.15,-0.25,-0.35,-0.45$ at $t=3.0$. The heat transport to upper parts of the domain for $C_{c o n v}>0$ is clearly seen. The adapted mesh for $C_{\text {conv }}=100$ at $t=3.0$ is shown in Fig. 12 . 


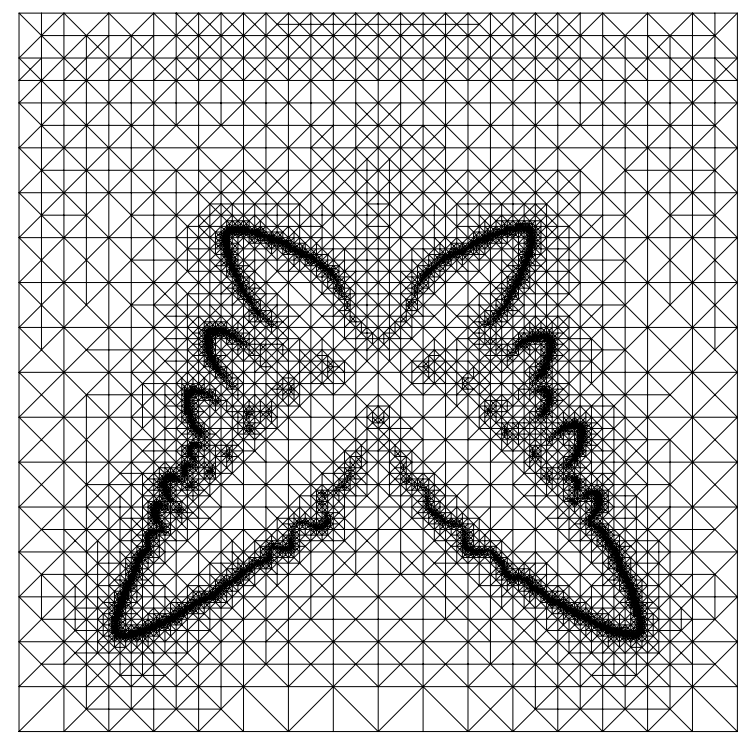

FIG. 12. Problem 4.1, $C_{c o n v}=100$; triangulation at $t=3.0$.

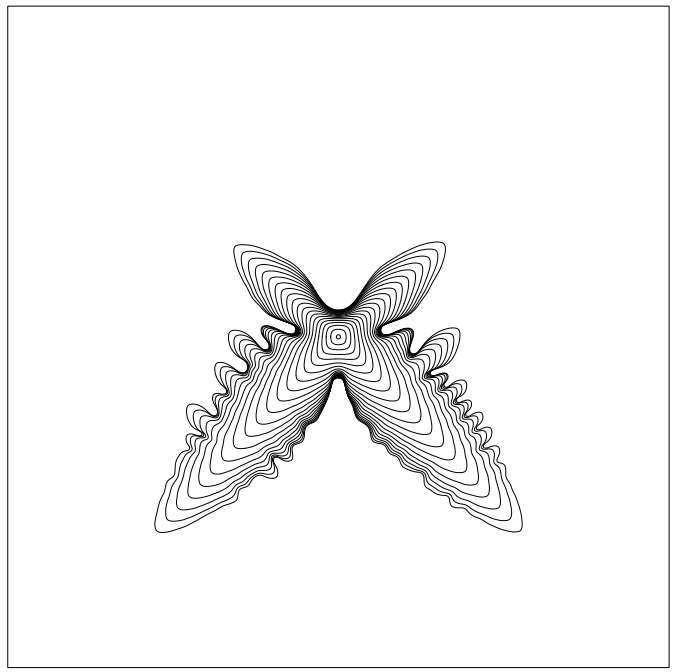

FIG. 13. Problem 4.2.1; interfaces after $0,10, \ldots, 200$ time steps.

\subsection{Transparent boundary conditions}

In order to reduce the influence of the Dirichlet boundary and approximate the problem in an unbounded domain, we impose natural boundary conditions for the coupled problem, i.e. absorbing boundary conditions for the flow problem and Neumann conditions for the temperature. To be more 


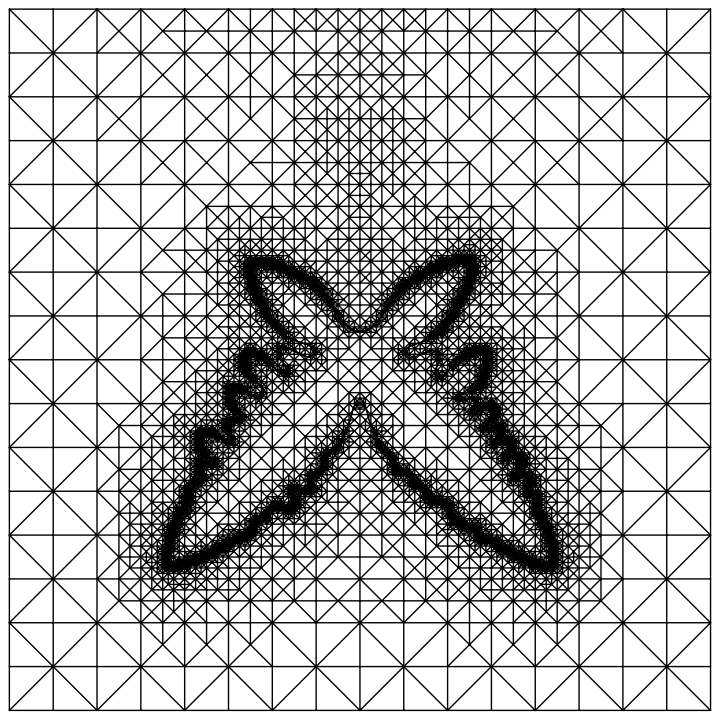

FIG. 14. Problem 4.2.1; triangulation at $t=2.0$.

precise, we impose the following condition for $u$ and $p$ :

$$
\frac{1}{\sqrt{G r}} \partial_{\nu} u-p v=g v \quad \text { on } \partial \Omega .
$$

Here, $g$ is a given function, accounting for the hydrostatic pressure and for a possibly horizontal pressure gradient for the case of a horizontal advection:

$$
g\left(x_{1}, x_{2}\right)=C_{1} x_{1}-\int_{0}^{x_{2}} \bar{\theta}\left(x_{1}, s\right) \mathrm{d} s
$$

with a constant $C_{1}\left(C_{1}=0\right.$ for the case of no horizontal advection) and $\bar{\theta}:=-0.5$ the 'main' part of $\theta$.

In the variational formulation for the momentum equation (17) turns out to be just the natural boundary condition for $u$ and $p$ at $\partial \Omega$ if the velocity space is defined such that the functions may have arbitrary values on $\partial \Omega$ and the right-hand side is given by

$$
F(\Phi):=\int_{\Omega_{l}} \vartheta e_{2} \cdot \Phi+\int_{\partial \Omega} g v \cdot \Phi,
$$

see also [17] for details.

For the temperature equation we impose a homogeneous Neumann condition on $\partial \Omega$, which means that the normal heat flux $q \cdot v=\left(-\frac{1}{P r \sqrt{G r}} \nabla \vartheta+C_{\text {conv } \vartheta u}\right) \cdot v$ on $\partial \Omega$ is given by the convective flux only: $q \cdot v=C_{\operatorname{con} v} \vartheta u \cdot v$. Again, defining the temperature accordingly, the Neumann condition is the natural boundary condition for the variational formulation.

Note that all subsequent simulations use the convection parameter $C_{\text {conv }}=100$. 


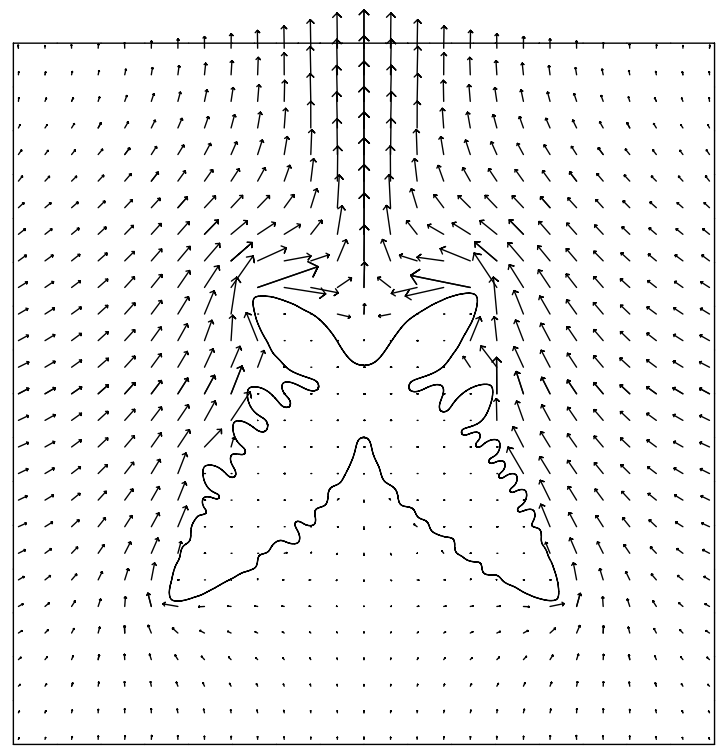

FIG. 15. Problem 4.2.1; interface and velocity at $t=2.0$.

4.2.1 Natural convection problem. Figures 13-16 show a problem similar to the third Dirichlet case, with only natural convection, i.e. $C_{1}=0$ in (18). Since the boundary condition (17) mimics an infinite domain, there are no convection rolls as in the previous examples and the transport is mainly upward.

4.2.2 Problem with additional advection. Figures 17-20 show a problem with additional horizontal advection, i.e. $C_{1}=0.2$. The influence of the advection on the crystal growth is again clearly visible, resulting in a fast and strongly non-symmetric growth.

\section{REFERENCES}

1. Ananth, R. \& GiLl, W. N. Dendritic growth with convection. J. Cryst. Growth 91, (1988) 587-598.

2. AnAnth, R. \& GiLl, W. N. Self-consistent theory of dendritic growth with convection. J. Cryst. Growth 108, (1991) 173-189.

3. B̈̈NSCH, E. Local mesh refinement in 2 and 3 dimensions. Impact Comput. Sci. Eng. No. 3, (1991) 181-191.

4. B̈̈NSCH, E. Adaptive finite-element techniques for Navier-Stokes equations and other transient problems. In Adaptive Finite and Boundary Elements. Computational Mechanics Publication's, Boston, co-published with Elsevier Applied Science, London (1993).

5. BÄNSCH, E. Simulation of instationary, incompressible flows. Acta Math. Univ. Comenianae 67, (1998) $101-114$.

6. BÄNsch, E. \& SChMidt, A. A finite element method for dendritic growth. In TAYlor, J. E. (ed.), Computational Crystal Growers Workshop, AMS Selected Lectures in Mathematics, pp. 16-20, Providence, RI. American Mathematical Society (1992). 


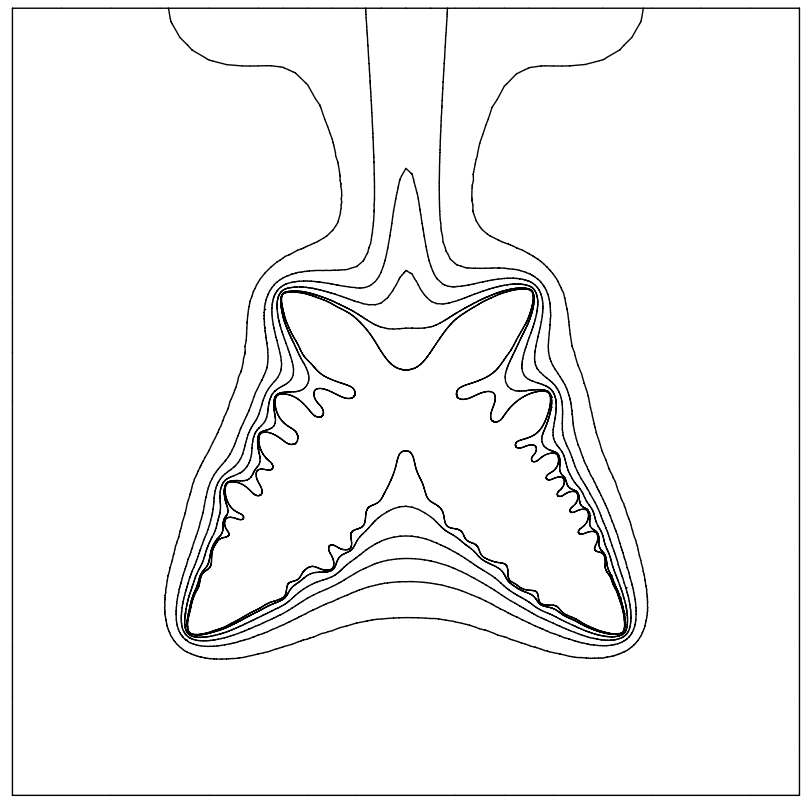

FIG. 16. Problem 4.2.1; interface and isothermal lines at $t=2.0$.

7. Bristeau, M. Glowinski, R., \& Periaux, J. Numerical methods for the Navier-Stokes equations. Application to the simulation of compressible and incompressible flows. Comput. Phys. Rep. 6, (1987) $73-188$

8. CAntor, B. \& Vogel, A. Dendritic solidification and fluid flow. J. Cryst. Growth 41, (1977) 109-123.

9. DZIUK, G. An algorithm for evolutionary surfaces. Numer. Math. 58, (1991) 603-611.

10. ERIKSSON, K. \& JOHNSON, C. Adaptive finite element methods for parabolic problems I: A linear model problem. SIAM J. Numer. Anal. 28, (1991) 43-77.

11. Glicksman, M. E. \& HuAng, S. C. Convective heat transfer during dendritic growth. In Zierep J. \& ORTEL, (eds), Convective Transport and Instability Phenomena, pp. 557-574. Braun, Karlsruhe (1982).

12. Glicksman, M. E., Koss, M. B., \& Winsa, E. A. Dendritic growth velocities in microgravity. Phys. Rev. Lett. 73, (1994) 573-576.

13. Glicksman, M. E. \& Marsh, S. P. The dendrite, in Handbook of Cryst. Growth, D. T. J. Hurle, ed., vol. 1, North-Holland, Amsterdam, 1993.

14. Glowinski, R., Pan, T.-W., Kearsley, A., \& PeriauX, J. Numerical simulation and optimal shape for viscous flow by a fictitious domain method. Int. J. Numer. Methods Fluids 20, (1995) 695-711.

15. Glowinski, R., Pan, T.-W., \& PeriauX, J. A fictitious domain method for external incompressible viscous flow modeled by Navier-Stokes equations. Comput. Methods Appl. Mech. Eng. 112, (1994) $133-148$.

16. Griebel, M., Merz, W., \& Neunhoeffer, T. Mathematical modelling and numerical simulation of freezing processes of a supercooled melt under consideration of density changes. Preprint 513, SFB 256, Bonn (1997).

17. Heywood, J. G., RAnNACHER, R., \& TUREK, S. Artificial boundaries and flux and pressure conditions for the incompressible Navier-Stokes equations. Int. J. Numer. Methods Fluids 22, (1996) 325-352. 


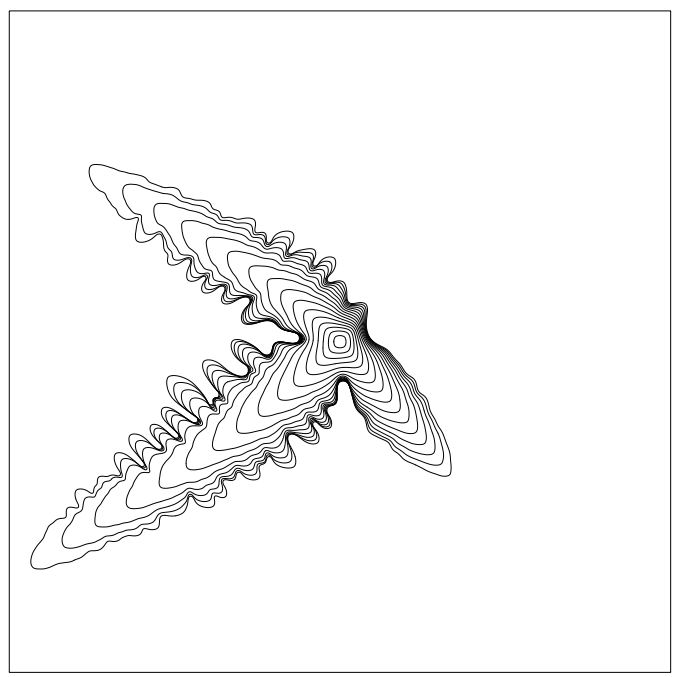

FIG. 17. Problem 4.2.2; interfaces after 7, 17, ., 157 time steps.

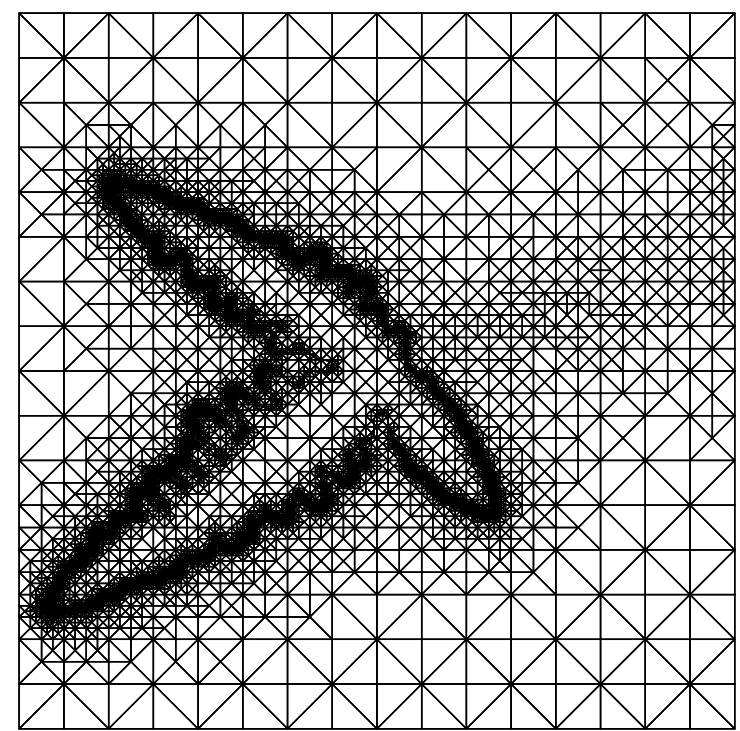

FIG. 18. Problem 4.2.2; triangulation at $t=1.57$.

18. LANGer, J. S. Instabilities and pattern formation in crystal growth. Rev. Mod. Phys. 52, (1980) 1-28.

19. Mitchell, W. A comparison of adaptive refinement techniques for elliptic problems. ACM Trans. Math. Softw. 15, (1989) 326-347.

20. Schmidt, A. Computation of three dimensional dendrites with finite elements. J. Comput. Phys. 125, (1996) 293-312.

21. SchmidT, A. Approximation of crystalline dendrite growth in two space dimensions. Acta Math. Univ. 


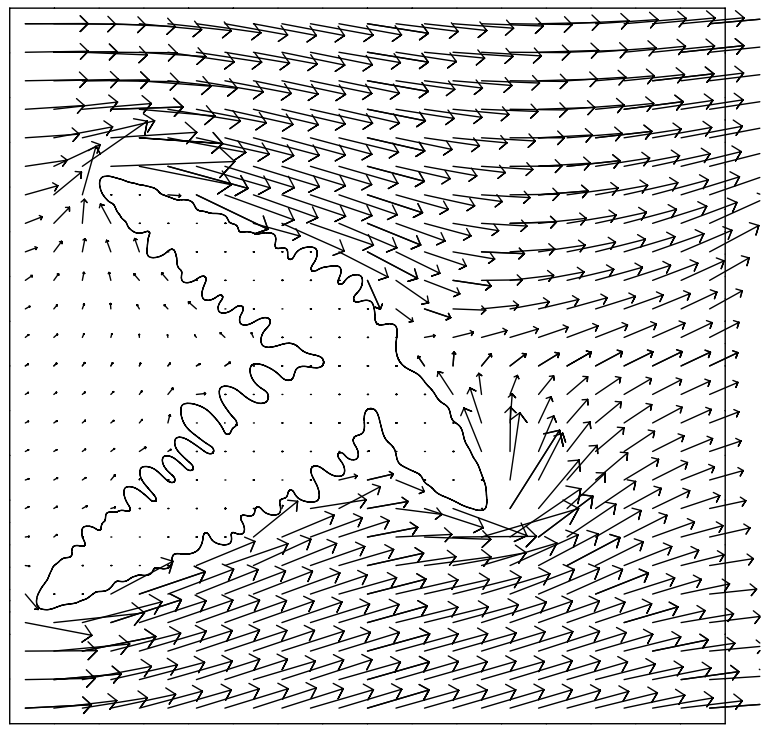

FIG. 19. Problem 4.2.2; interface and velocity at $t=1.57$.

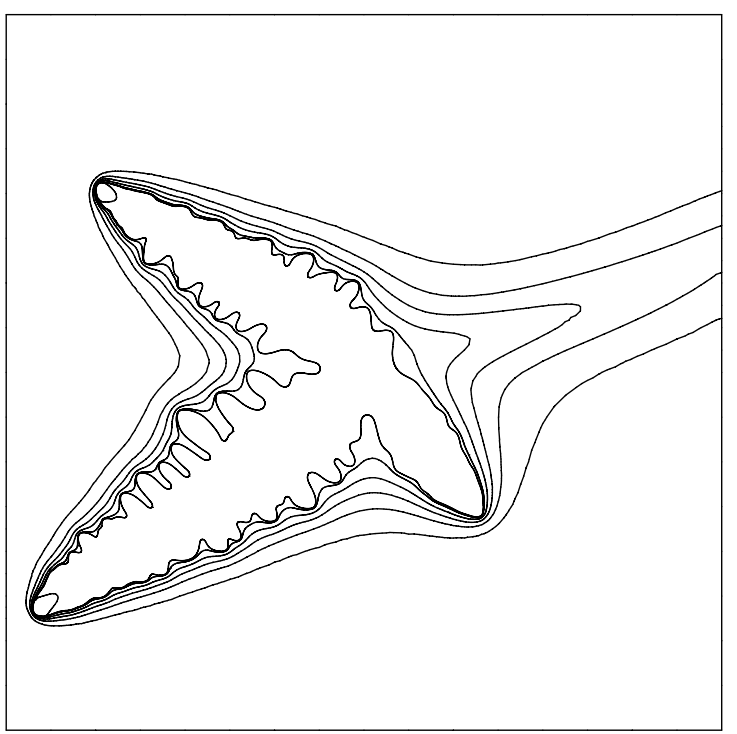

FIG. 20. Problem 4.2.2; interface and isothermal lines at $t=1.57$.

Comenianae 67, (1998) 57-68.

22. VEESER, A. Fehlerabschätzungen für ein Verfahren zur Berechnung von zweidimensionalen Dendriten. $\mathrm{PhD}$ thesis, Universität Freiburg (1998).

23. VEESER, A. Error estimates for semi-discrete dendritic growth. Interfaces and Free Boundaries 1, (1999) 227-255. 


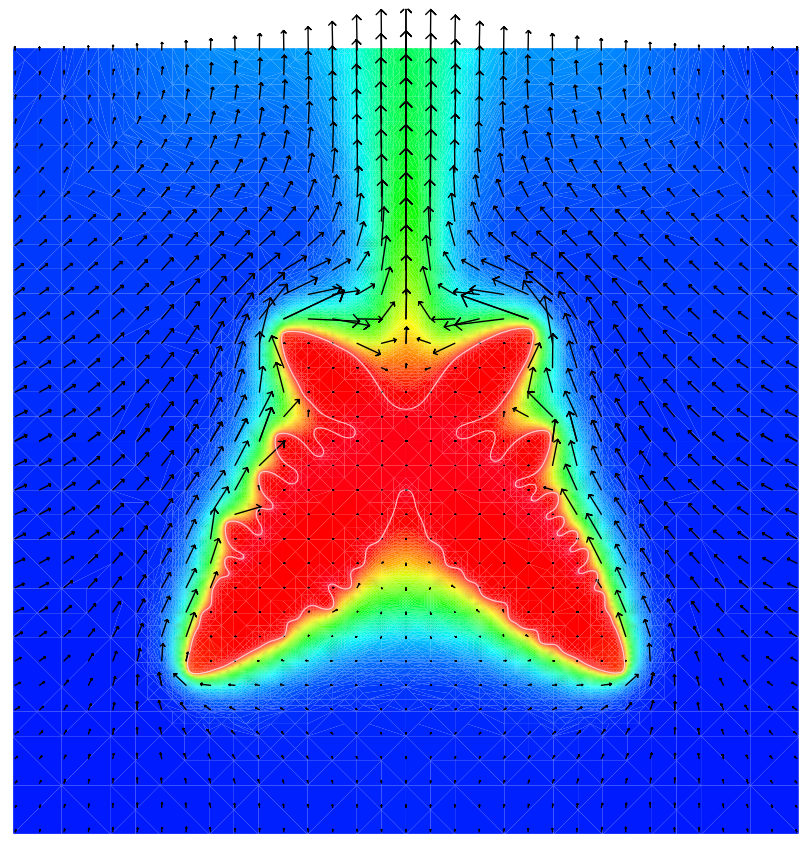

Color plate 1: Problem 4.2.1; temperature, velocity, and interface at time $t=2.0$.

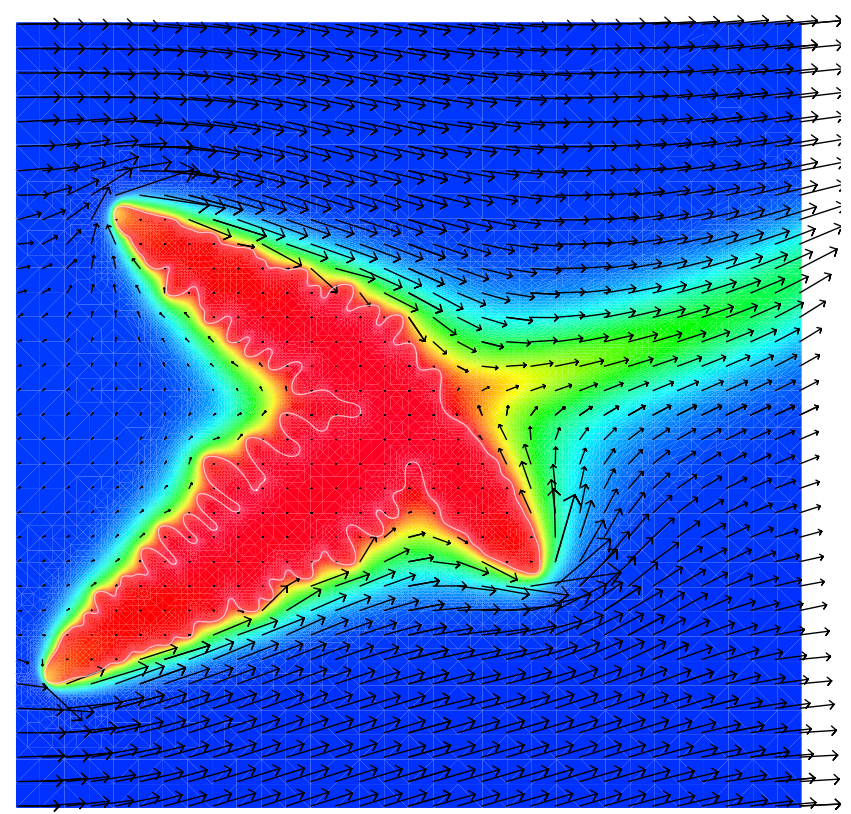

Color plate 2: Problem 4.2.2; temperature, velocity, and interface at time $t=1.57$. 